\title{
Analysis for Transverse Sensitivity of the Microaccelerometer
}

\author{
Yu LIU ${ }^{1,2,3}$, Guochao WANG ${ }^{1,2,3}$, Changwen GUO ${ }^{1,2,3}$ \\ ${ }^{1}$ Automobile College, Chongqing University of Technology, Chongqing, China \\ ${ }^{2}$ Key Laboratory of Automobile Parts \& Test Technique in Chongqing, Chongqing, China \\ ${ }^{3}$ Chongqing Engineering Research Center for Automobile Power System and Control, Chongqing, China \\ E-mail: liuyu_cq@126.com \\ Received January 10, 2009; revised February 21, 2009; accepted February 23, 2009
}

\begin{abstract}
For the microaccelerometer, strong axial response and weak cross-axial one are always expected. This paper presents a general analysis about transverse sensitivity of the microaccelerometer. The analysis model is developed, where the influence of response stiffness and damping in different axes, as well as symmetrical decline angles of 3 degrees of freedom system is considered. Moreover, multi-freedom vibration equations based on the analysis model are established. And the equations are solved on condition that damping force is ignored. Finally, the theoretical analysis about transverse sensitivity is accomplished, and some effective methods, which are beneficial to reduce cross disturbance, are provided.
\end{abstract}

Keywords: MEMS, Accelerometers, Transverse Sensitivity, Multi-Freedom Vibration Equation

\section{Introduction}

For the microaccelerometer, there should be no output if the input acceleration is along the cross axis. In fact, however, the output created by forces induced in orthogonal axis is not equal to zero. This phenomena is called cross coupling, which is measured by transverse sensitivity [1-3].

In this paper, the analysis model for cross disturbance of the microaccelerometer is developed, where the influence of response stiffness and damping in different axes, as well as symmetrical decline angles of 3 degrees of freedom system is considered. Moreover, multi- freedom vibration equations based on the analysis model are established. And the equations are solved on condition that damping force is ignored. Finally, the theoretical analysis about transverse sensitivity is accomplished, and some effective methods, which are beneficial to reduce cross disturbance, are provided.

\section{Transverse Sensitivity}

Transverse sensitivity is the ratio of the output caused by acceleration perpendicular to the main sensitivity axis divided by the basic sensitivity in the main direction. It is an important characteristic of the microaccelerometer, and is primarily caused by two factors [4-6]. One is from the inherent microstructure, which may be eliminated by adopting the appropriate working principle and optimizing the design parameters. The other is from inaccuracies in fabrication process, package orientation and misalignment, which is only to be reduced as possible as we can.

For example, $x$-axis accelerometer, due to inevitability of errors in fabrication and misalignments, the applied acceleration can be expressed as acceleration along the $x$-axis and accelerations perpendicular to the main sensitivity axis, denoted as $\vec{a}_{x}, \vec{a}_{y}, \vec{a}_{z}$ respectively. Therefore, the output is given by

$$
V_{\text {xout }}=S_{x} a_{x}+S_{y x} a_{y}+S_{z x} a_{z}
$$

where $S_{y x}$ or $S_{z x}$ is transverse sensitivity of $x$-axis in $y$ or $z$ direction. Unfortunately, the accelerometer cannot distinguish the change in voltage caused by accelerations $\vec{a}_{y}$ and $\vec{a}_{z}$, which results in a difference of $S_{y x} a_{y}+S_{z x} a_{z}$.

Disturbance and coupling from different axes have important influences on the performance of the microaccelerometer. So strong axial response and weak crossaxial one are always expected. And the transverse sensitivity is always expected to small enough, even close to zero. 


\section{Analysis of Transverse Sensitivity}

\subsection{Model}

In most cases, the microstructure of the accelerometer can be represented as a mass-spring-damper system. Figure 1 shows the mechanical model of the microaccelerometer with a single $x$ - degree of freedom. In perfect condition, elastic deformation of the spring induced by the inertial force is always along the $x$-axis no matter where acceleration signal is from. In fact, however, the phenomenon of cross coupling exists inevitably. On the one hand, the elastic deformation of the equivalent spring occurs not only in primary $x$-axis but also in orthogonal $y$-axis and $z$-axis, and on the other hand, the displacement of the microstructure under acceleration $\vec{a}_{x}$ is not always along the primary $x$-axis, which may be at an angle with the ideal sensitive axis [7].

Figure 2 illustrates the mechanical model of microaccelerometer with three degrees of freedom, where elastic deformation is along $x$-axis, $y$-axis and $z$-axis. So the acceleration ponderance $\vec{a}_{x}, \vec{a}_{y}, \vec{a}_{z}$ are detected by the corresponding degree of freedom system. Because the microstructure has the same proof mass, the different equivalent stiffness and damping coefficients, denoted as $K_{x}, K_{y}, K_{z}$ and $B_{x}, B_{y}, B_{z}$ respectively, the model in Figure 2 is the analysis model of cross disturbance resulted from stiffness and damping in different axes.

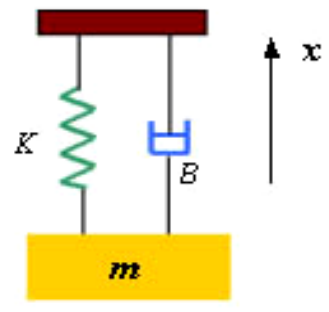

Figure 1. Simplified mechanical model of the microaccelerometer with a single $x$ - degree of freedom.

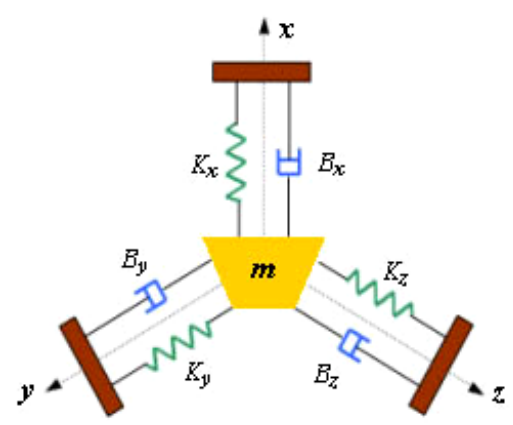

Figure 2. Analysis model of cross disturbance resulted from stiffness and damping in different axes.
Figure 3 shows the other model of the microaccelerometer, where the spring and the damper are not along the primary axis but that at an angle with the corresponding ideal axis. For example, $x$ - degree of freedom system, as illustrated in Figure 3(a), due to inevitability of errors in fabrication process, package orientation as well as misalignment, the spring $K_{x}$ and damper $B_{x}$ are all at an angle with $x$-axis, which is called symmetrical decline angle anddenoted as $\alpha_{x}$. At the same time, the spring $K_{x}$ and damper $B_{x}$ are also at an angle with $y$-axis or $z$-axis, denoted as $\beta_{x}, \gamma_{x}$ respectively. Most often, $\alpha_{x}$ is quite small, and $\beta_{x}, \gamma_{x}$ are all close to $\pi / 2$. Therefore,

$$
\cos ^{2} \alpha_{x}+\cos ^{2} \beta_{x}+\cos ^{2} \gamma_{x}=1
$$

Similarly, $\beta_{y}, \gamma_{z}$ are the symmetrical decline angles of $y$ - and $z$ - degree of freedom systems respectively, as shown in Figure 3 (b) and (c).

So the model in Figure 3 is the analysis model of cross disturbance resulted from the symmetrical decline angles.

\subsection{Solution}

In order to analyze the influence of cross disturbance, the multi-freedom vibration equations based on the abovementioned models should be established.

Here sinusoidal signal is considered. $a_{x} \sin \omega t$, $a_{y} \sin \omega t, a_{z} \sin \omega t$ denote three projections of vector acceleration respectively. Furthermore, assume the displacement function is as follows:

$$
\left\{\begin{array}{l}
w_{x}=W_{x}^{(x)} \sin \omega t \\
w_{y}=W_{y}^{(x)} \sin \omega t \\
w_{z}=W_{z}^{(x)} \sin \omega t
\end{array}\right.
$$

where $W_{x}^{(x)}, W_{y}^{(x)}, W_{z}^{(x)}$ are the amplitudes along the $x, y$ and $z$-direction respectively.

For $x$-degree of freedom system, the vibration equation responded to acceleration signal $a_{x} \sin \omega t$ is given by:

$$
m\left\{\begin{array}{l}
\ddot{w}_{x} \\
\ddot{w}_{y} \\
\ddot{w}_{z}
\end{array}\right\}+\left[\begin{array}{ccc}
B_{x x} & B_{x y} & B_{x z} \\
B_{x y} & B_{y y} & B_{y z} \\
B_{x z} & B_{y z} & B_{z z}
\end{array}\right]\left\{\begin{array}{l}
\dot{w}_{x} \\
\dot{w}_{y} \\
\dot{w}_{z}
\end{array}\right\}+\left[\begin{array}{ccc}
K_{x x} & K_{x y} & K_{x z} \\
K_{x y} & K_{y} & K_{y z} \\
K_{x z} & K_{y z} & K_{z z}
\end{array}\right]\left\{\begin{array}{l}
w_{x} \\
w_{y} \\
w_{z}
\end{array}\right\}=\left\{\begin{array}{l}
m a_{x} \sin \omega t \\
0 \\
0
\end{array}\right\}
$$

where $w_{x}, w_{y}, w_{z}$ are the displacements of proof mass along the $x, y$ and $z$-direction respectively. $\dot{w}_{x}, \dot{w}_{y}, \dot{w}_{z}$ and $\ddot{w}_{x}, \ddot{w}_{y}, \ddot{w}_{z}$ denote the first and the second derivative of displacement $w_{x}, w_{y}, w_{z}$ with respect to time $t$ respectively. $K_{x x}, K_{y y}, K_{z z}$ are the self-stiffness of equiva- 


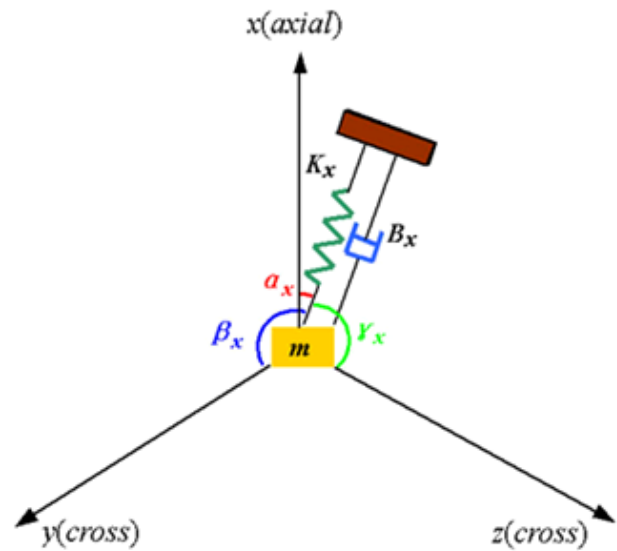

(a)

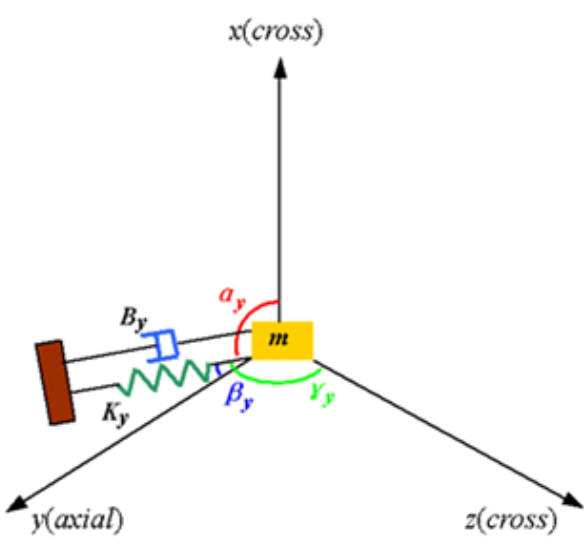

(b)

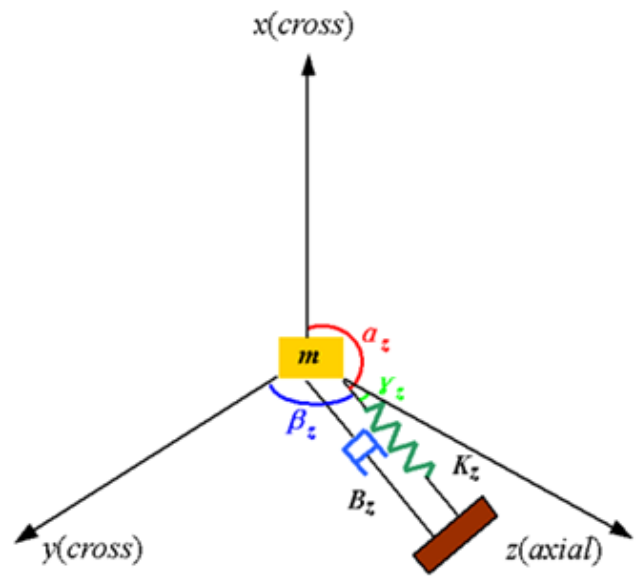

(c)

Figure 3. Analysis model of cross disturbance resulted from the symmetrical decline angles. (a) $x$-degree of freedom system. (b) $y$-degree of freedom system. (c) $z$-degree of freedom system.

lent spring $K_{x}$, which reflect responsibility of spring $K_{x}$ in three orthogonal axes. $K_{x y}, K_{y z}, K_{x z}$ are the coupling stiffness of equivalent spring $K_{x}$, which reflect responsibility of spring $K_{x}$ in three coupling orthogonal axes. And $B_{x x}$, $B_{y y}, B_{z z}$ are the self-damping of equivalent coefficient $B_{x}$, which reflect the damping effect of $B_{x}$ in three orthogonal axes. $B_{x y}, B_{y z}, B_{x z}$ are the coupling damping of equivalent coefficient $B_{x}$, which reflect the damping effect of $B_{x}$ in three coupling orthogonal axes.

Substituting Equation (3) into Equation (4), we get the system of three linear equations in three variables $W_{x}^{(x)}$, $W_{y}^{(x)}, W_{z}^{(x)}$ :

$$
\left\{\begin{array}{l}
\left(K_{x x} \sin \omega t+B_{x x} \omega \cos \omega t-m \omega^{2} \sin \omega t\right) W_{x}^{(x)}+\left(K_{x y} \sin \omega t+B_{x y} \omega \cos \omega t\right) W_{y}^{(x)}+\left(K_{x z} \sin \omega t+B_{x z} \omega \cos \omega t\right) W_{z}^{(x)}=m a_{x} \sin \omega t \\
\left(K_{x y} \sin \omega t+B_{x y} \omega \cos \omega t\right) W_{x}^{(x)}+\left(K_{y y} \sin \omega t+B_{y y} \omega \cos \omega t-m \omega^{2} \sin \omega t\right) W_{y}^{(x)}+\left(K_{y z} \sin \omega t+B_{y z} \omega \cos \omega t\right) W_{z}^{(x)}=0 \\
\left(K_{x z} \sin \omega t+B_{x z} \omega \cos \omega t\right) W_{x}^{(x)}+\left(K_{y z} \sin \omega t+B_{y z} \omega \cos \omega t\right) W_{y}^{(x)}+\left(K_{z z} \sin \omega t+B_{z z} \omega \cos \omega t-m \omega^{2} \sin \omega t\right) W_{z}^{(x)}=0
\end{array}\right.
$$

Usually, there are three ponderances of vector acceleration, denoted as $\vec{a}_{x}, \vec{a}_{y}, \vec{a}_{z}$. They are responded by the respective degree of freedom system. Furthermore, response along the $x, y$ and $z$-direction exist in each degree of freedom system. So there are nine amplitude expressions responded to the acceleration signal. In order to simplify the analysis, damping force is ignored. Therefore, Equation (5) is simplified to: 
$\left\{\begin{array}{l}\left(K_{x x}-m \omega^{2}\right) W_{x}^{(x)}+K_{x y} W_{y}^{(x)}+K_{x z} W_{z}^{(x)}=m a_{x} \\ K_{x y} W_{x}^{(x)}+\left(K_{y y}-m \omega^{2}\right) W_{y}^{(x)}+K_{y z} W_{z}^{(x)}=0 \\ K_{x z} W_{x}^{(x)}+K_{y z} W_{y}^{(x)}+\left(K_{z z}-m \omega^{2}\right) W_{z}^{(x)}=0\end{array}\right.$

Hence,

$$
\left\{\begin{array}{l}
W_{x}^{(x)}=-\frac{K_{x} \sin ^{2} \alpha_{x}-m \omega^{2}}{\left(K_{x}-m \omega^{2}\right) \omega^{2}} a_{x} \\
W_{y}^{(x)}=\frac{K_{x} \cos \alpha_{x} \cos \beta_{x}}{\left(K_{x}-m \omega^{2}\right) \omega^{2}} a_{x} \\
W_{z}^{(x)}=\frac{K_{x} \cos \alpha_{x} \cos \gamma_{x}}{\left(K_{x}-m \omega^{2}\right) \omega^{2}} a_{x}
\end{array}\right.
$$

Likewise, the amplitudes responded to acceleration signal $a_{y} \sin \omega t, a_{z} \sin \omega t$ are respectively given by

$$
\begin{aligned}
& \left\{\begin{array}{l}
W_{x}^{(y)}=\frac{K_{y} \cos \alpha_{y} \cos \beta_{y}}{\left(K_{y}-m \omega^{2}\right) \omega^{2}} a_{y} \\
W_{y}^{(y)}=-\frac{K_{y} \sin ^{2} \beta_{y}-m \omega^{2}}{\left(K_{y}-m \omega^{2}\right) \omega^{2}} a_{y} \\
W_{z}^{(y)}=\frac{K_{y} \cos \beta_{y} \cos \gamma_{y}}{\left(K_{y}-m \omega^{2}\right) \omega^{2}} a_{y} \\
W_{x}^{(z)}=\frac{K_{z} \cos \alpha_{z} \cos \gamma_{z}}{\left(K_{z}-m \omega^{2}\right) \omega^{2}} a_{z} \\
W_{y}^{(z)}=\frac{K_{z} \cos \beta_{z} \cos \gamma_{z}}{\left(K_{z}-m \omega^{2}\right) \omega^{2}} a_{z} \\
W_{z}^{(z)}=-\frac{K_{z} \sin ^{2} \gamma_{z}-m \omega^{2}}{\left(K_{z}-m \omega^{2}\right) \omega^{2}} a_{z}
\end{array}\right.
\end{aligned}
$$

\subsection{Analysis}

Building on the previous analysis, we get the nine amplitude expressions responded to the sinusoidal acceleration signal, as listed in Table 1.

For 3-axis microaccelerometer, what we need is the amplitude response in leading diagonal of Table 1 . They should be the strongest, whereas the others are the cross disturbance. Therefore, transverse sensitivity of $x$-axis in $y$ - and $z$-direction are given by

$$
\begin{aligned}
& S_{y x}=\frac{S_{y \text {-cross }}}{S_{x-\text { axial }}}=\frac{K_{y} \cos \alpha_{y} \cos \beta_{y}}{m \omega^{2}-K_{x} \sin ^{2} \alpha_{x}} \cdot \frac{K_{x}-m \omega^{2}}{K_{y}-m \omega^{2}}, \\
& S_{z x}=\frac{S_{z \text {-cross }}}{S_{x-\text { axial }}}=\frac{K_{z} \cos \alpha_{z} \cos \gamma_{z}}{m \omega^{2}-K_{x} \sin ^{2} \alpha_{x}} \cdot \frac{K_{x}-m \omega^{2}}{K_{z}-m \omega^{2}} .
\end{aligned}
$$

Similarly,

$$
\begin{aligned}
& S_{x y}=\frac{S_{x-\text { cross }}}{S_{y \text {-axial }}}=\frac{K_{x} \cos \alpha_{x} \cos \beta_{x}}{m \omega^{2}-K_{y} \sin ^{2} \beta_{y}} \cdot \frac{K_{y}-m \omega^{2}}{K_{x}-m \omega^{2}}, \\
& S_{z y}=\frac{S_{z \text {-cross }}}{S_{y \text {-axial }}}=\frac{K_{z} \cos \beta_{z} \cos \gamma_{z}}{m \omega^{2}-K_{y} \sin ^{2} \beta_{y}} \cdot \frac{K_{y}-m \omega^{2}}{K_{z}-m \omega^{2}} . \\
& S_{x z}=\frac{S_{x-\text { cross }}}{S_{z \text {-axial }}}=\frac{K_{x} \cos \alpha_{x} \cos \gamma_{x}}{m \omega^{2}-K_{z} \sin ^{2} \gamma_{z}} \cdot \frac{K_{z}-m \omega^{2}}{K_{x}-m \omega^{2}}, \\
& S_{y z}=\frac{S_{y-\text { cross }}}{S_{z-\text { axial }}}=\frac{K_{y} \cos \beta_{y} \cos \gamma_{y}}{m \omega^{2}-K_{z} \sin ^{2} \gamma_{z}} \cdot \frac{K_{z}-m \omega^{2}}{K_{y}-m \omega^{2}} .
\end{aligned}
$$

If the microaccelerometer sensitive to the change in displacement has three primary axes, then the equation $K_{x}=K_{y}=K_{z}$ is always expected for uniform sensitivity in three sense directions. So the expressions of transverse sensitivity in Equation (10)-(12) could be simplified. Considering constraints for symmetrical decline angles of $x$-, $y$ - and $z$-degrees of freedom system, that is, $\alpha_{x}, \beta_{y}, \gamma_{z}$ are all close to zero, it's not difficult to find that transverse sensitivity only relates to the coupling angles and the way for small transverse sensitivity is to make the coupling angles equal to $\pi / 2$. Of course, $\alpha_{y}, \alpha_{z}$ are the coupling angles of $x$-system, while $\beta_{x}, \beta_{z}$ and $\gamma_{x}, \gamma_{y}$ are those of $y$ and $z$-systems respectively. If the coupling angles are all equal to $\pi / 2$, then transverse sensitivity $S=0$.

It should be pointed out if the microaccelerometer has only one or two primary axes, then the above six expressions about transverse sensitivity will be decreased by four or two. For instance, there are only $S_{y x}, S_{z x}$ for $x$-axis accelerometer. So transverse sensitivity relates not only to the coupling angles but also to the response stiffness in different axes. Therefore, two methods are recommended to reduce transverse sensitivity. One is to make the coupling angles equal to $\pi / 2$. The other is to ensure the response stiffness in the primary axis far less than that in the cross axis.

\section{Summary}

This paper presents a general analysis about transverse sensitivity of the microaccelerometer. Firstly, the analysis model for cross disturbance of the microaccelerometer is developed, where the influence of response stiffness and damping in different axes, as well as symmetrical decline angles of 3 degrees of freedom system are considered. Secondly, multi-freedom vibration equations based on the analysis model are established. And the equations are solved on condition that damping force is ignored. Finally, some effective methods, which are beneficial to reduce cross disturbance, are provided. For the microacceleromesensitive to the change in displacement, if it has 
Table 1. Amplitude responded to the sinusoidal acceleration signal.

\begin{tabular}{|c|c|c|c|}
\hline Acceleration & $x$-direction & $y$-direction & $z$-direction \\
\hline$a_{x} \sin \omega t$ & $-\frac{K_{x} \sin ^{2} \alpha_{x}-m \omega^{2}}{\left(K_{x}-m \omega^{2}\right) \omega^{2}} a_{x}$ & $\frac{K_{x} \cos \alpha_{x} \cos \beta_{x}}{\left(K_{x}-m \omega^{2}\right) \omega^{2}} a_{x}$ & $\frac{K_{x} \cos \alpha_{x} \cos \gamma_{x}}{\left(K_{x}-m \omega^{2}\right) \omega^{2}} a_{x}$ \\
\hline$a_{y} \sin \omega t$ & $\frac{K_{y} \cos \alpha_{y} \cos \beta_{y}}{\left(K_{y}-m \omega^{2}\right) \omega^{2}} a_{y}$ & $-\frac{K_{y} \sin ^{2} \beta_{y}-m \omega^{2}}{\left(K_{y}-m \omega^{2}\right) \omega^{2}} a_{y}$ & $\frac{K_{y} \cos \beta_{y} \cos \gamma_{y}}{\left(K_{y}-m \omega^{2}\right) \omega^{2}} a_{y}$ \\
\hline$a_{z} \sin \omega t$ & $\frac{K_{z} \cos \alpha_{z} \cos \gamma_{z}}{\left(K_{z}-m \omega^{2}\right) \omega^{2}} a_{z}$ & $\frac{K_{z} \cos \beta_{z} \cos \gamma_{z}}{\left(K_{z}-m \omega^{2}\right) \omega^{2}} a_{z}$ & $-\frac{K_{z} \sin ^{2} \gamma_{z}-m \omega^{2}}{\left(K_{z}-m \omega^{2}\right) \omega^{2}} a_{z}$ \\
\hline
\end{tabular}

three primary axes, then transverse sensitivity only relates to the coupling angle and the way for small transverse sensitivity is to make the coupling angles equal to $\pi / 2$. If it has one or two primary axes, then transverse sensitivity relates not only to the coupling angle but also to the response stiffness in different axes. So in order to reduce transverse sensitivity, two methods are recommended. One is to make the coupling angles equal to $\pi / 2$. The other is to ensure the response stiffness in the primary axis far less than that in the cross axis.

\section{References}

[1] L. Fei, X. X. Zhong, Z. Y. Wen, et al, "Methods for reducing sensitivity of micromachined silicon accelerometer," Optics and Precision Engineering, No. 3, pp. 64-68, 1995

[2] J. S. Wang, Q. Wang, and S. H. Sun, "Effect of crosscoupling coefficient of accelerometer on gyros-free iner tial measurement unit," Journal of Chinese Inertial Technology, No. 11, pp. 29-33, 2003.

[3] L. Zhong., J. G. Liu, and H. Y. Zhao, "A solution to the problem of excessive error of piezoelectrical accelerometer's cross axis sensitivity," Electro-Mechanical Engineering, Vol. 20, pp. 4-5, 2004.

[4] Y Liu, Z. Y. Wen, Z. Q. Wen, et al, "Design and fabrication of a high-sensitive capacitive biaxial microaccelerometer," J. Micromech. Microeng, Vol. 17, pp. 36-41, 2007.

[5] Y Liu, Z. Y. Wen, and H. Y. Yang, "Effect of fabrication characteristic of 2-d microaccelerometer on performance," Journal of Functional Materials and Devices, Vol. 14, pp. 331-335, 2008.

[6] Y. Liu, Z. Y. Wen, L. Q. Zhang, et al, "Structure design and system simulation of 2-d microaccelerometer," ISTM05, Vol. 3, pp. 2071-2075.

[7] N. Zeng, "Research on the key technology of fiber optic accelerometers," Doctor Thesis, Tsinghua University, pp. 97-100, 2005 